Kragujevac Journal of Mathematics

Volume 44(4) (2020), PAges 551-562.

\title{
LOWER BOUNDS FOR INVERSE SUM INDEG INDEX OF GRAPHS
}

\author{
I. GUTMAN ${ }^{1}$, M. MATEJIĆ ${ }^{2}$, E. MILOVANOVIĆ ${ }^{2}$, AND I. MILOVANOVIĆ $^{2}$
}

Abstract. Let $G=(V, E), V=\{1,2, \ldots, n\}$, be a simple connected graph with $n$ vertices and $m$ edges and let $d_{1} \geq d_{2} \geq \cdots \geq d_{n}>0$, be the sequence of its vertex degrees. With $i \sim j$ we denote the adjacency of the vertices $i$ and $j$ in $G$. The inverse sum indeg index is defined as $I S I=\sum \frac{d_{i} d_{j}}{d_{i}+d_{j}}$ with summation going over all pairs of adjacent vertices. We consider lower bounds for $I S I$. We first analyze some lower bounds reported in the literature. Then we determine some new lower bounds.

\section{INTRODUCTION}

Let $G=(V, E), V=\{1,2, \ldots, n\}, E=\left\{e_{1}, e_{2}, \ldots, e_{m}\right\}$, be a simple connected graph with $n$ vertices and $m$ edges, and let $\Delta=d_{1} \geq d_{2} \geq \cdots \geq d_{n}=\delta>0$, $d_{i}=d(i)$, and $d\left(e_{1}\right) \geq d\left(e_{2}\right) \geq \cdots \geq d\left(e_{m}\right)$, be sequences of its vertex and edge degrees, respectively. We denote by $\Delta_{e_{1}}=d\left(e_{1}\right)+2$ and $\delta_{e_{1}}=d\left(e_{m}\right)+2$. If the vertices $i$ and $j$ are adjacent, we write $i \sim j$.

In graph theory, an invariant is a property of graphs that depends only on their abstract structure, not on the labeling of vertices or edges, or on the drawing of the graph. Such quantities are also referred to as topological indices. Topological indices gained considerable popularity because of their applications in chemistry as molecular structure descriptors $[2,24,25]$.

An important class of graph invariants are those whose general formula is

$$
V D B=V D B(G)=\sum_{i \sim j} \Phi\left(d_{i}, d_{j}\right)
$$

Key words and phrases. Degree (of vertex), degree (of edge), inverse sum indeg index, Zagreb index.

2010 Mathematics Subject Classification. Primary: 05C12. Secondary: 05C50.

DOI 10.46793/KgJMat2004.551G

Received: March 09, 2018.

Accepted: June 28, 2018. 
which are usually referred to as vertex-degree based topological indices. Here $\Phi$ may be any function satisfying the condition $\Phi(x, y)=\Phi(y, x)$. A very large number of particular VDB indices has been considered in the literature, some of which are listed below. There are countless papers reporting relations for VDB indices, which includes bounds (in terms of various graph parameters), characterization of graphs extremal w.r.t. some particular VDB index (in some particular class of graphs), and inequalities between various members of the VDB family. Readers interested in this topic may consult the recent collections of review articles [12-14].

The present paper contributes to the theory of VDB indices, comparing some previously known inequalities and challenging their validity, and offering a few new results of the same kind.

The oldest VDB topological indices, the first and the second Zagreb indices are defined as (see $[8,9])$

$$
M_{1}=M_{1}(G)=\sum_{i=1}^{n} d_{i}^{2} \quad \text { and } \quad M_{2}=M_{2}(G)=\sum_{i \sim j} d_{i} d_{j},
$$

where the first Zagreb index can be expressed as

$$
M_{1}=\sum_{i \sim j}\left(d_{i}+d_{j}\right) .
$$

Bearing in mind that for the edge $e$ connecting the vertices $i$ and $j$,

$$
d(e)=d_{i}+d_{j}-2,
$$

the index $M_{1}$ can also be considered as an edge-degree based invariant (see [17])

$$
M_{1}=\sum_{i=1}^{m}\left[d\left(e_{i}\right)+2\right] .
$$

A so-called forgotten topological index is defined as (see [8])

$$
F=F(G)=\sum_{i=1}^{n} d_{i}^{3}=\sum_{i \sim j}\left(d_{i}^{2}+d_{j}^{2}\right)
$$

It can be easily observed that for the indices $M_{2}$ and $F$ the following identities hold:

$$
F+2 M_{2}=\sum_{i=1}^{m}\left[d\left(e_{i}\right)+2\right]^{2} \text { and } F-2 M_{2}=\sum_{i \sim j}\left(d_{i}-d_{j}\right)^{2} .
$$

Multiplicative versions of the first and second Zagreb indices, denoted by $\Pi_{1}$ and $\Pi_{2}$, respectively, were first considered in a paper [10] published in 2011, and were promptly followed by numerous additional studies. These indices are defined as:

$$
\Pi_{1}=\Pi_{1}(G)=\prod_{i=1}^{n} d_{i}^{2} \quad \text { and } \quad \Pi_{2}=\Pi_{2}(G)=\prod_{i \sim j} d_{i} d_{j} .
$$


One year later, motivated by the identity (1.1), the multiplicative sum-Zagreb index was conceived as [3]:

$$
\Pi_{1}^{*}=\Pi_{1}^{*}(G)=\prod_{i \sim j}\left(d_{i}+d_{j}\right) .
$$

Probably the most popular and most thoroughly investigated molecular-structure descriptor is the classical Randić (or connectivity) index

$$
R=R(G)=\sum_{i \sim j} \frac{1}{\sqrt{d_{i} d_{j}}}
$$

invented by Randić in 1975 [21].

Replacing in (1.2) multiplication by summation, the so-called sum-connectivity index was put forward as (see [32])

$$
S C I=S C I(G)=\sum_{i \sim j} \frac{1}{\sqrt{d_{i}+d_{j}}} .
$$

In [1] (see also $[11,16]$ ) a topological index called general Randić index, $R_{\alpha}$, was introduced as

$$
R_{\alpha}=R_{\alpha}(G)=\sum_{i \sim j}\left(d_{i} d_{j}\right)^{\alpha}
$$

where $\alpha$ is an arbitrary real number. For $\alpha=-1 / 2$ we have $R=R_{-1 / 2}$, whereas for $\alpha=1 / 2$, the reciprocal Randic index, $R R,[11,16]$ is obtained.

In order to improve the predictive power of the Randić index, a large number of additional vertex-degree based topological descriptors was introduced. The geometricarithmetic index, introduced in [30], is defined as

$$
G A=G A(G)=\sum_{i \sim j} \frac{2 \sqrt{d_{i} d_{j}}}{d_{i}+d_{j}} .
$$

The harmonic index, introduced in [4], is defined as

$$
H=H(G)=\sum_{i \sim j} \frac{2}{d_{i}+d_{j}} .
$$

It should be noted that $\Pi_{1}^{*}, S C I$, and $H$ can be considered as edge-degree based topological indices as well, since the following identities hold:

$$
\Pi_{1}^{*}=\prod_{i=1}^{m}\left[d\left(e_{i}\right)+2\right], \quad S C I=\sum_{i=1}^{m} \frac{1}{\sqrt{d\left(e_{i}\right)+2}}, \quad H=\sum_{i=1}^{m} \frac{2}{d\left(e_{i}\right)+2} .
$$

In a series of papers [26-28,31], Vukičević introduced the so-called Adriatic indices, providing a general method for constructing vertex-degree based graph invariants; for review see [29]. Vukičević himself restricted the considerations to some 148 such 
indices, although their possible number would be infinite. One of these Adriatic indices, named symmetric division deg index, is

$$
S D D=S D D(G)=\sum_{i \sim j} \frac{1}{2}\left(\frac{d_{i}}{d_{j}}+\frac{d_{j}}{d_{i}}\right) .
$$

Another Adriatic index, the so-called inverse sum indeg index, was singled out in [26] as being a significantly accurate predictor of total surface area of octane isomers. It is defined as

$$
I S I=I S I(G)=\sum_{i \sim j} \frac{d_{i} d_{j}}{d_{i}+d_{j}}
$$

In this paper, we are interested in lower bounds on $I S I$. We first perform the analysis of some earlier reported lower bounds for $I S I[5,19,23]$. Then we determine some new lower bounds for it, in terms of some other vertex-degree based graph invariants.

\section{Preliminary CONSIDERAtions}

In this section, we analyze some lower bounds for the inverse sum indeg index reported in $[5,19,23]$.

In $[23]$ the following inequality was proven

$$
I S I \geq \frac{(n-1)^{2}}{n},
$$

with equality if and only if $G \cong K_{1, n-1}$. This bound is the best possible in its class.

In [5] it was proven

$$
I S I \geq \frac{m^{2}}{n}
$$

with equality if and only if the graph $G$ is regular or biregular. This bound depends on the parameters $n$ and $m$, and it is the best one in its class, so far.

The bounds given by (2.1) and (2.2), although simple, are very important and, as we shall demonstrate, are convenient for testing whether other lower bounds, depending on some other parameters, have any sense. Of course, it is of interest to determine other (lower) bounds that establish relationships between ISI and other graph invariants. But, if these inequalities are weaker than inequalities (2.1) and (2.2), the question of their purpose arises. In that sense we will analyze lower bounds for ISI obtained in [5] and [19].

In [5] the following lower bounds for ISI were also established:

$$
\begin{aligned}
& I S I \geq \frac{m^{2} \delta^{2}}{M_{1}}, \\
& I S I \geq \frac{\delta^{2} H}{2},
\end{aligned}
$$




$$
\begin{aligned}
& I S I \geq \frac{M_{2}}{2 \Delta}, \\
& I S I \geq \frac{\delta^{2}(S C I)^{2}}{m},
\end{aligned}
$$

$I S I \geq H$

$$
I S I \geq \frac{M_{1}}{2}-\frac{F}{4 \delta},
$$$$
I S I \geq \frac{m^{2} \sqrt{\delta \Delta}}{(\delta+\Delta) R}
$$$$
I S I \geq \frac{(S C I)^{2}}{R_{-1}},
$$

$$
I S I \geq m\left(\frac{\Pi_{2}}{\Pi_{1}^{*}}\right)^{1 / m}
$$

whereas in [19] it was proven that

$$
I S I \geq \frac{\sqrt{\delta \Delta} H M_{2}}{m(\delta+\Delta)} .
$$

The inequalities (2.3)-(2.12) are all correct. However, it is questionable whether any of the bounds given by (2.3)-(2.10) are worthy. In what follows we discuss this matter.

Since

$$
M_{1}=\sum_{i=1}^{n} d_{i}^{2} \geq n \delta^{2}
$$

we have that

$$
\frac{m^{2}}{n} \geq \frac{m^{2} \delta^{2}}{M_{1}} .
$$

Thus, the inequality (2.3) is a direct consequence of the inequality (2.2).

Since

$$
\frac{n \delta^{2} H}{2}=\frac{\delta^{2}}{2} \sum_{i \sim j}\left(\frac{1}{d_{i}}+\frac{1}{d_{j}}\right) \sum_{i \sim j} \frac{2}{d_{i}+d_{j}} \leq \frac{\delta^{2}}{2} \frac{2 m}{\delta} \frac{m}{\delta}
$$

it holds

$$
\frac{m^{2}}{n} \geq \frac{\delta^{2} H}{2} .
$$

Thus, the inequality (2.4) is a direct consequence of the inequality (2.2). 
Using the arithmetic-harmonic mean inequality for real numbers (see for example [18]), we get

$$
\frac{1}{2} H M_{1}=\frac{1}{2} \sum_{i \sim j} \frac{2}{d_{i}+d_{j}} \sum_{i \sim j}\left(d_{i}+d_{j}\right) \geq m^{2}
$$

that is

$$
\frac{\delta^{2} H}{2} \geq \frac{m^{2} \delta^{2}}{M_{1}}
$$

implying that the inequality (2.3) is a consequence of (2.4).

If $m \geq n$, the inequality (2.5) is a consequence of (2.2).

Let $m=n-1$, i.e., $G$ is a tree. In [6] it was proven that

$$
M_{2}(T) \leq \Delta(2 n-\Delta-1-k)+k(k-1),
$$

where

$$
k \equiv n-1 \quad(\bmod \Delta-1), \quad 1 \leq k \leq n-1 .
$$

From (2.13) it follows

$$
M_{2}(T) \leq \Delta(2 n-\Delta-1-k)+k(k-1) \leq \frac{2 \Delta(n-1)^{2}}{n}
$$

wherefrom we get

$$
\frac{m^{2}}{n}=\frac{(n-1)^{2}}{n} \geq \frac{M_{2}(T)}{2 \Delta}
$$

This means that the inequality (2.5) is a consequence of (2.2) for every connected graph $G$.

According to the inequality

$$
(S C I)^{2}=\left(\sum_{i \sim j} \frac{1}{\sqrt{d_{i}+d_{j}}}\right)^{2} \leq m \sum_{i \sim j} \frac{1}{d_{i}+d_{j}}=\frac{m H}{2},
$$

it follows

$$
\frac{m^{2}}{n} \geq \frac{\delta^{2} H}{2} \geq \frac{\delta^{2}(S C I)^{2}}{m}
$$

This means that the inequality (2.6) is a consequence of both (2.2) and (2.4).

Let $m=n-1$, i.e., $G$ is a tree of order $n$, and let $n \geq 3$. Then $d_{i}+d_{j} \geq 3$ for every $i \sim j$. Therefore,

$$
\frac{(n-1)^{2}}{n} \geq \frac{2}{3}(n-1) \geq H
$$

It follows that in this case the inequality (2.7) is a consequence of both (2.1) and (2.2).

Let $m \geq n$. Then $d_{i}+d_{j} \geq 2$ for every $i \sim j$. Then we have

$$
\frac{m^{2}}{n} \geq m \geq H \text {. }
$$

Therefore, in this case, the inequality (2.7) is also a consequence of (2.2).

The inequality (2.2) is stronger than the inequality (2.8) when $G$ is a biregular graph, or $G \cong P_{n}$, or $G \cong K_{n}-e$, or $G \cong K_{n-1}+e$. When $n \geq 3$ and $G$ is not a 
regular graph, then we could not find any connected graph for which the inequality (2.8) is stronger than the inequality (2.2). Moreover, if $\Delta \geq 2 \delta$, then the right-hand side of (2.8) can be negative. Therefore, the right-hand side of (2.8) should be avoided when estimating lower bound for ISI.

Since

$$
n=\sum_{i \sim j}\left(\frac{1}{d_{i}}+\frac{1}{d_{j}}\right)=\sum_{i \sim j} \frac{d_{i}+d_{j}}{d_{i} d_{j}}=\sum_{i \sim j} \frac{d_{i}+d_{j}}{\sqrt{d_{i} d_{j}}} \frac{1}{\sqrt{d_{i} d_{j}}}
$$

and

$$
\frac{d_{i}+d_{j}}{\sqrt{d_{i} d_{j}}}=\sqrt{\frac{d_{i}}{d_{j}}}+\sqrt{\frac{d_{j}}{d_{i}}} \leq \sqrt{\frac{\Delta}{\delta}}+\sqrt{\frac{\delta}{\Delta}},
$$

for every edge in the graph $G$, it follows

$$
n \leq \frac{(\Delta+\delta) R}{\sqrt{\Delta \delta}} .
$$

Therefore,

$$
\frac{m^{2}}{n} \geq \frac{m^{2} \sqrt{\Delta \delta}}{(\Delta+\delta) R}
$$

Thus, the inequality (2.9) is a consequence of the inequality (2.2).

The inequality (2.2) is stronger than the inequality (2.10) when $G \cong P_{n}$, or $G \cong$ $K_{n}-e$ or $G \cong K_{n-1}+e, n \geq 3$. If $n \geq 3$ and $G$ is not a regular or biregular graph, then we could not find any connected graph for which the inequality (2.10) is stronger than the inequality (2.2). However, it remains an open question whether this is the case for every connected graph under given conditions.

The inequality (2.11) is stronger than the inequality (2.2) for $G \cong P_{n}, G \cong K_{n}-e$ or $G \cong K_{n-1}+e$. Again, we could not find any connected graph which is not regular or biregular for which the inequality (2.2) is stronger than the inequality (2.11). It is still an open question if this is always the case.

The inequalities (2.2) and (2.12) are not comparable. Thus, for example, if the connected graph is biregular or $G \cong K_{n-1}+e$, then the inequality (2.2) is stronger than the inequality (2.12). If, however, $G \cong P_{n}$ or $G \cong K_{n}-e$, then the inequality (2.12) is stronger than (2.2).

\section{Main Results}

Before we establish some new lower bounds for $I S I$, we recall some discrete inequalities for real number sequences that will be used subsequently.

Let $p=\left(p_{i}\right)$ and $a=\left(a_{i}\right), i=1,2, \ldots, m$, be positive real number sequences with the properties $p_{1}+p_{2}+\cdots+p_{m}=1$ and $0<a \leq a_{i} \leq A<+\infty$. In [22] the following inequality was proven

$$
\sum_{i=1}^{m} p_{i} a_{i}+a A \sum_{i=1}^{m} \frac{p_{i}}{a_{i}} \leq a+A
$$


Equality holds if and only if $a_{i}=A$ or $a_{i}=a$, for every $i=1,2, \ldots, m$.

Let $x=\left(x_{i}\right)$ and $a=\left(a_{i}\right), i=1,2, \ldots, m$, be positive real number sequences. In [20] it was proven that for any $r \geq 0$ holds

$$
\sum_{i=1}^{m} \frac{x_{i}^{r+1}}{a_{i}^{r}} \geq \frac{\left(\sum_{i=1}^{m} x_{i}\right)^{r+1}}{\left(\sum_{i=1}^{m} a_{i}\right)^{r}}
$$

with equality if and only if $\frac{a_{1}}{x_{1}}=\cdots=\frac{a_{m}}{x_{m}}$.

If $a=\left(a_{i}\right), i=1,2, \ldots, m$, is a positive real number sequence, then [15]

$$
\left(\sum_{i=1}^{m} \sqrt{a_{i}}\right)^{2} \geq \sum_{i=1}^{m} a_{i}+m(m-1)\left(\prod_{i=1}^{m} a_{i}\right)^{1 / m} .
$$

Equality holds if and only if $a_{1}=a_{2}=\cdots=a_{m}$.

Theorem 3.1. Let $G$ be a simple connected graph. Then

$$
I S I \geq \frac{4 R_{-1} M_{2}+\Delta_{e_{1}} \delta_{e_{1}} H^{2}}{4\left(\Delta_{e_{1}}+\delta_{e_{1}}\right) R_{-1}} .
$$

Equality holds if and only if $G$ is regular or biregular.

Proof. For $p_{i}:=\frac{d_{i} d_{j}}{\left(d_{i}+d_{j}\right) I S I}, a_{i}:=d_{i}+d_{j}, a=\delta_{e_{1}}, A=\Delta_{e_{1}}$, where summation is performed over all pairs of adjacent vertices of $G$, the inequality (3.1) becomes

$$
\sum_{i \sim j} d_{i} d_{j}+\Delta_{e_{1}} \delta_{e_{1}} \sum_{i \sim j} \frac{d_{i} d_{j}}{\left(d_{i}+d_{j}\right)^{2}} \leq\left(\Delta_{e_{1}}+\delta_{e_{1}}\right) I S I,
$$

i.e.,

$$
M_{2}+\Delta_{e_{1}} \delta_{e_{1}} \sum_{i \sim j} \frac{d_{i} d_{j}}{\left(d_{i}+d_{j}\right)^{2}} \leq\left(\Delta_{e_{1}}+\delta_{e_{1}}\right) I S I .
$$

For $r=1, x_{i}:=\frac{1}{d_{i}+d_{j}}, a_{i}:=\frac{1}{d_{i} d_{j}}$, where summation goes over all pairs of adjacent vertices, the inequality (3.2) transforms into

$$
\sum_{i \sim j} \frac{d_{i} d_{j}}{\left(d_{i}+d_{j}\right)^{2}} \geq \frac{\left(\sum_{i \sim j} \frac{1}{d_{i}+d_{j}}\right)^{2}}{\sum_{i \sim j} \frac{1}{d_{i} d_{j}}},
$$

that is

$$
\sum_{i \sim j} \frac{d_{i} d_{j}}{\left(d_{i}+d_{j}\right)^{2}} \geq \frac{H^{2}}{4 R_{-1}} .
$$

In view of (3.5) and (3.6), we obtain (3.4). 
The equality in (3.6) holds if and only if for any two pairs of adjacent vertices $i \sim j$ and $u \sim v$

$$
\frac{1}{d_{i}}+\frac{1}{d_{j}}=\frac{1}{d_{u}}+\frac{1}{d_{v}} .
$$

Let $j$ and $u$ be two vertices adjacent to $i$, that is $i \sim j$ and $i \sim u$. Then, from the above identity, it follows $d_{j}=d_{u}$. Since $G$ is a connected graph, equality in (3.6) holds if and only if $G$ is regular or biregular.

Equality in (3.5) holds if and only if $d_{i}+d_{j}=\Delta_{e_{1}}$ or $d_{i}+d_{j}=\delta_{e_{1}}$, for every edge of $G$. This means that equality in (3.5) holds if and only if $G$ is regular or biregular or for some edges $d_{i}+d_{j}=\Delta_{e_{1}}$ holds whereas for the remaining edges $d_{i}+d_{j}=\delta_{e_{1}}$. This means that equality in (3.4) holds if and only if $G$ is regular or biregular.

In the next theorem we obtain a lower bound for $I S I$ in terms of the parameters $m, \Delta_{e_{1}}, \delta_{e_{1}}$, and the topological indices $M_{2}$ and $S D D$.

Theorem 3.2. Let $G$ be a simple connected graph with $m$ edges. Then

$$
I S I \geq \frac{2 M_{2}(S D D+m)+m^{2} \Delta_{e_{1}} \delta_{e_{1}}}{2(S D D+m)\left(\Delta_{e_{1}}+\delta_{e_{1}}\right)} .
$$

Equality is attained if and only if for any two pairs of adjacent vertices $i \sim j$ and $u \sim v$ the identity

$$
\frac{d_{i}}{d_{j}}+\frac{d_{j}}{d_{i}}=\frac{d_{u}}{d_{v}}+\frac{d_{v}}{d_{u}}
$$

holds.

Proof. By the arithmetic-harmonic mean inequality (see e.g. [18]), we have

$$
\sum_{i \sim j} \frac{d_{i} d_{j}}{\left(d_{i}+d_{j}\right)^{2}} \sum_{i \sim j} \frac{\left(d_{i}+d_{j}\right)^{2}}{d_{i} d_{j}} \geq m^{2} .
$$

Since

$$
\sum_{i \sim j} \frac{\left(d_{i}+d_{j}\right)^{2}}{d_{i} d_{j}}=\sum_{i \sim j} \frac{d_{i}^{2}+d_{j}^{2}+2 d_{i} d_{j}}{d_{i} d_{j}}=\sum_{i \sim j} \frac{d_{i}^{2}+d_{j}^{2}}{d_{i} d_{j}}+2 m=2(S D D+m),
$$

from (3.10) and the above it follows

$$
\sum_{i \sim j} \frac{d_{i} d_{j}}{\left(d_{i}+d_{j}\right)^{2}} \geq \frac{m^{2}}{2(S D D+m)} .
$$

From this and inequality (3.5) we obtain (3.8).

Equality in (3.10) is attained if and only if for any two pairs of adjacent vertices $i \sim j$ and $u \sim v$ the equality (3.9) holds. Consequently, equality in (3.8) holds if and only if for any two pairs of adjacent vertices $i \sim j$ and $u \sim v$ the equality (3.9) is valid. 
In the following theorem we determine a lower bound for $I S I$ in terms of the parameters $m, \Delta_{e_{1}}, \delta_{e_{1}}$, and the topological indices $M_{2}$ and $G A$.

Theorem 3.3. Let $G$ be a simple connected graph with $m$ edges. Then

$$
I S I \geq \frac{4 m M_{2}+\Delta_{e_{1}} \delta_{e_{1}}(G A)^{2}}{4 m\left(\Delta_{e_{1}}+\delta_{e_{1}}\right)} .
$$

Equality in (3.11) holds if and only if for any two pairs of adjacent vertices $i \sim j$ and $u \sim v$, the equality (3.9) is valid.

Proof. Since

$$
\sum_{i \sim j} \frac{d_{i} d_{j}}{\left(d_{i}+d_{j}\right)^{2}}=\sum_{i \sim j}\left(\frac{\sqrt{d_{i} d_{j}}}{d_{i}+d_{j}}\right)^{2} \geq \frac{1}{m}\left(\sum_{i \sim j} \frac{\sqrt{d_{i} d_{j}}}{d_{i}+d_{j}}\right)^{2}
$$

it follows

$$
\sum_{i \sim j} \frac{d_{i} d_{j}}{\left(d_{i}+d_{j}\right)^{2}} \geq \frac{1}{m}\left(\frac{G A}{2}\right)^{2} .
$$

From this inequality and (3.5) we obtain (3.11).

The equality case in Theorem 3.3 is proved in a same way as in the case of Theorem 3.2 .

In the following theorem we determine a lower bound for $I S I$ in terms of $M_{1}$ and $R R$.

Theorem 3.4. Let $G$ be a simple connected graph with $m$ edges. Then

$$
I S I \geq \frac{(R R)^{2}}{M_{1}}
$$

Equality holds if and only if for any two pairs of adjacent vertices $i \sim j$ and $u \sim v$, the equality (3.9) is valid.

Proof. For $r=1, x_{i}:=\sqrt{d_{i} d_{j}}, a_{i}:=d_{i}+d_{j}$, where summation goes over all pairs of adjacent vertices of $G$, the inequality (3.2) transforms into

$$
\sum_{i \sim j} \frac{\left(\sqrt{d_{i} d_{j}}\right)^{2}}{d_{i}+d_{j}} \geq \frac{\left(\sum_{i \sim j} \sqrt{d_{i} d_{j}}\right)^{2}}{\sum_{i \sim j}\left(d_{i}+d_{j}\right)}
$$

that is

$$
I S I \geq \frac{(R R)^{2}}{M_{1}}
$$

The equality case in (3.12) is proved in a same way as in the case of Theorem 3.2. 
The inequalities (3.4), (3.8), (3.11) and (3.12) are stronger than the inequality (2.2) when $G \cong P_{n}, G \cong K_{n}-e$ or $G \cong K_{n-1}+e$. We could not find any connected graph for which the inequality (2.2) is stronger than these inequalities. However, it is an open question whether these inequalities are always stronger than (2.2).

\section{REFERENCES}

[1] B. Bollobás and P. Erdő̋, Graphs of extremal weights, Ars Combin. 50 (1998), 225-233.

[2] J. Devillers and A. T. Balaban (Eds.), Topological Indices and Related Descriptors in QSAR and QSPR, Gordon \& Breach, New York, 1999.

[3] M. Eliasi, A. Iranmanesh and I. Gutman, Multiplicative versions of first Zagreb index, MATCH Commun. Math. Comput. Chem. 68 (2012), 217-230.

[4] S. Fajtlowicz, On conjectures on Graffiti-II, Congr. Numer. 60 (1987), 187-197.

[5] F. Falahati-Nezhad, M. Azari and T. Došlić, Sharp bounds on the inverse sum indeg index, Discrete Appl. Math. 217 (2017), 185-195.

[6] C. M. Fonseca and D. Stevanović, Further properties of the second Zagreb index, MATCH Commun. Math. Comput. Chem. 72 (2014), 655-668.

[7] B. Furtula and I. Gutman, A forgotten topological index, J. Math. Chem. 53 (2015), 1184-1190.

[8] I. Gutman and N. Trinajstić, Graph theory and molecular orbitals. Total $\pi$-electron energy of alternant hydrocarbons, Chem. Phys. Lett. 17 (1972), 535-538.

[9] I. Gutman, B. Ruščić, N. Trinajstić and C. F. Wilcox, Graph theory and molecular orbitals. XII. Acyclic polyenes, J. Chem. Phys. 62 (1975), 3399-3405.

[10] I. Gutman, Multiplicative Zagreb indices of trees, Bull. Int. Math. Virtual Inst. 1 (2011), 13-19.

[11] I. Gutman and B. Furtula (Eds.), Recent Results in the Theory of Randić Index, University of Kragujevac, Kragujevac, 2008.

[12] I. Gutman, B. Furtula, K. C. Das, E. Milovanović and I. Milovanović (Eds.), Bounds in Chemical Graph Theory - Basics, University of Kragujevac, Kragujevac, 2017.

[13] I. Gutman, B. Furtula, K. C. Das, E. Milovanović and I. Milovanović (Eds.), Bounds in Chemical Graph Theory - Mainstreams, University of Kragujevac, Kragujevac, 2017.

[14] I. Gutman, B. Furtula, K. C. Das, E. Milovanović and I. Milovanović (Eds.), Bounds in Chemical Graph Theory - Advances, University of Kragujevac, Kragujevac, 2017.

[15] H. Kober, On the arithmetic and geometric means and on Hölder's inequality, Proc. Amer. Math. Soc. 9 (1958), 452-459.

[16] X. Li and I. Gutman, Mathematical Aspects of Randić-Type Molecular Structure Descriptors, University of Kragujevac, Kragujevac, 2006.

[17] I. Ž. Milovanović, E. I. Milovanović, I. Gutman and B. Furtula, Some inequalities for the forgotten topological index, International Journal of Applied Graph Theory 1 (2017), 1-15.

[18] D. S. Mitrinović and P. M. Vasić, Analytic Inequalities, Springer, Berlin, 1970.

[19] K. Pattabiraman, Inverse sum indeg index of graphs, AKCE Int. J. Graphs Comb. (to appear).

[20] J. Radon, Theorie und Anwendungen der Absolut Additiven Mengenfunktionen, Sitzungsber. Acad. Wissen. Wien 122, 1913, 1295-1438.

[21] M. Randić, On characterization of molecular branching, J. Amer. Chem. Soc. 97 (1975), 66096615.

[22] B. C. Rennie, On a class of inequalities, J. Aust. Math. Soc. 3 (1963), 442-448.

[23] J. Sedlar, D. Stevanović and A. Vasilyev, On the inverse sum indeg index, Discrete Appl. Math. 184 (2015), 202-212.

[24] R. Todeschini and V. Consonni, Handbook of Molecular Descriptors, Wiley-VCH, Weinheim, 2000.

[25] R. Todeschini and V. Consonni, Molecular Descriptors for Chemoinformatics, Wiley-VCH, Weinheim, 2009. 
[26] D. Vukičević, Bond additive modeling 2. Mathematical properties of max-min rodeg index, Croat. Chem. Acta 83 (2010), 261-273.

[27] D. Vukičević, Bond additive modeling 4. QSPR and QSAR studies of variable adriatic indices, Croat. Chem. Acta 84 (2011), 87-91.

[28] D. Vukičević, Bond additive modeling 5. Mathematical properties of the variable sum exdeg index, Croat. Chem. Acta 84 (2011), 93-101.

[29] D. Vukičević, Bond additive modeling. Adriatic indices - overview of results, in: I. Gutman, B. Furtula (Eds.), Novel Molecular Structure Descriptors - Theory and Applications II, University of Kragujevac, Kragujevac, 2010, pp. 269-302.

[30] D. Vukičević and B. Furtula, Topological index based on the ratios of geometrical and arithmetical means of end-vertex degrees of edges, J. Math. Chem. 46 (2009), 1369-1376.

[31] D. Vukičević and M. Gašperov, Bond additive modeling 1. Adriatic indices, Croat. Chem. Acta 83 (2010), 243-260.

[32] B. Zhou and N. Trinajstić, On a novel connectivity index, J. Math. Chem. 46 (2009), 1252-1270.

${ }^{1}$ FACUlty OF SCIENCE,

UNIVERSITY OF KRAGUJEVAC,

Kragujevac, Serbia

Email address: gutman@kg.ac.rs

${ }^{2}$ Faculty of Electronic Engineering, UNIVERSITY OF Niš,

Niš, SERBIA

Email address: \{marjan.matejic, ema, igor\}@elfak.ni.ac.rs 